\title{
Čerenkov second-harmonic generation in the strong conversion limit: new effects
}

\author{
Gijs J. M. Krijnen and Hugo J. W. M. Hoekstra \\ Lightwave Devices Group, University of Twente, P.O. Box 217, 7500 AE, Enschede, The Netherlands
}

George I. Stegeman and William Torruellas

Center for Research and Education in Optics and Lasers, University of Central Florida, P.O. Box 162700, Orlando, Florida 32816-2700

Received November 30, 1995

Using beam propagation method calculations, we show that new effects occur for second-harmonic generation in the Cerenkov regime when it is optimized for efficient coupling to leaky modes. The fundamental throughput becomes independent of the input power over a few decades; i.e., optical power limiting is found. At even higher input powers, the fundamental throughput abruptly switches back to approximately the input power as a consequence of generating coupled second-harmonic-fundamental solitary waves in the waveguide because of the cascading effect. (c) 1996 Optical Society of America

Interest in $\chi^{(2)}$ materials and related nonlinear wave-propagation effects has been driven for a long time by potential applications to efficient blue-light generation by second-harmonic generation (SHG) and electro-optical switching. ${ }^{1}$ From a practical point of view this interest has been dominated by guided mode (fundamental) $\rightarrow$ guided mode (second-harmonic) interactions. ${ }^{2}$ Guided mode $\rightarrow$ radiation mode interactions, called Čerenkov SHG, have been studied as well, but analytical relations are difficult to obtain because of the problems associated with normalization of radiation modes, and consequently most research has focused on the low-depletion regime. ${ }^{3}$ The analysis of large conversion by means of Čerenkov SHG requires a special beam propagation code in which the calculation of the propagation of the fields at the two wavelengths is alternated with the calculation of the nonlinear interactions. ${ }^{4}$ Previously we used such a SHG-beam propagation method to show that leaky modes play a very important role in SHG processes in the Cerenkov regime. ${ }^{5-7}$ Here we explore the strong conversion Cerenkov regime in slab waveguides and show that some new and interesting effects occur. Specifically we show that there is a power range in which the fundamental output does not increase with the fundamental input corresponding to optical limiting. At high powers we find a sharp transition to high transmission of the fundamental because of the formation of solitary waves in the waveguide.

The geometry studied here is shown in Fig. 1. It was originally used to simulate an SF1 fiber whose core consisted of single-crystal 2-( $N, N$-dimethylamino) 5-nitroacetanilide (DAN), in which large SHG and nonlinear phase changes have been reported. ${ }^{6,8}$ An input power of $3.14 \times 10^{5} \mathrm{~W} / \mathrm{m}$ corresponds roughly to a total input power of $1 \mathrm{~W}$ in the equivalent fiber. A fundamental beam at $\omega(1.32 \mu \mathrm{m})$ is guided in a 5 -mm-long slab waveguide with cladding refractive indices of $n_{\mathrm{c} 1}(\omega)=1.6875$ and $n_{\mathrm{c} 1}(2 \omega)=1.7097$ and a $4-\mu \mathrm{m}$-thick film with indices $n_{c}(\omega)=1.6992$ and $n_{c}(2 \omega)=1.6976$ and a $\chi^{(2)}$ of $25 \mathrm{pm} / \mathrm{V}$. This waveguide does not support a guided mode at $2 \omega$. The Čerenkov condition that must be satisfied for the second-harmonic ( $\mathrm{SH}$ ) field to be radiated into the cladding is $2 \beta(\omega)>n_{\mathrm{c} 1}(2 \omega) 2 k_{0}$, where $\beta(\omega)=n_{\text {eff }}(\omega) k_{0}$ is the fundamental guided wave vector and $n_{\text {eff }}(\omega)$ is the effective index. This usually requires a larger index dispersion with frequency in the cladding than in the film. The radiation angle $\theta$ defined in Fig. 1 is given by $\cos (\theta)=n_{\text {eff }}(\omega) / n_{\mathrm{c} 1}(2 \omega)$. Note that the waveguide values were carefully chosen so that the SH couples optimally into the lowest-order leaky mode of the waveguide. This condition was shown previously to optimize both the Čerenkov SH emission and the SH trapped within the core of the fiber. ${ }^{5,7}$

In Plate I we show the evolution of the fundamental and the SH fields down the waveguide for three input powers. Note that different scales are used for the waveguide width and length and that the angle $\theta$ is only $\sim 7^{\circ}$. At low input powers (case I, Plate I, top) the radiative loss into the $\mathrm{SH}$ is small and occurs almost uniformly along the full length of the waveguide. The relatively strong SHG right at the input is caused by an interface between a linear and a nonlinear medium. At intermediate powers (case II,
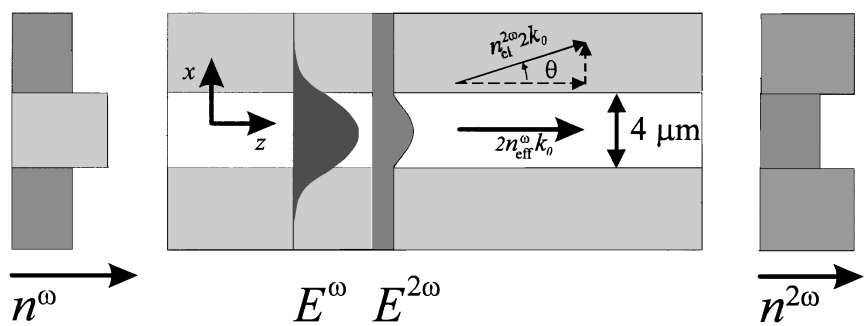

Fig. 1. Schematic of the numerically investigated structure. The waveguide is $5 \mathrm{~mm}$ long, has a thickness of $4 \mu \mathrm{m}$, and has refractive-index profiles as drawn schematically for $\omega$ and $2 \omega$ at the left-hand and the right-hand sides, respectively.

(C) 1996 Optical Society of America 
Plate I, middle) the conversion into $\mathrm{SH}$ occurs near the waveguide input, and only a small fraction of the fundamental power is transmitted to the waveguide output. The situation changes dramatically at high powers (case III, Plate I, bottom). Minimal SH is radiated into the cladding, and the fundamental and the harmonic travel together inside the fiber. Close examination of this last case indicates that the two beams are actually confined inside the waveguide boundaries. The large distinction between the three regimes is also very well illustrated by Fig. 2, which shows the overlap of the fundamental field with the $\mathrm{TE}_{0}(\omega)$ mode (in the low-power limit) as a function of propagation distance. Case I displays the classical Čerenkov SHG, involving modest conversion and negligible depletion of the fundamental. In case II there is a rapid decline in the fundamental power with distance, followed by a slower rate of depletion. Additionally some fluctuations in the mode power, indicating periodic power exchange between the fundamental and the SH fields, can be observed at small propagation distances. In case III the fundamental field stabilizes at a more-or-less fixed value in a very short distance, after which there is only a very small ripple in the overlap.

Here we are interested primarily in the fundamental power transmitted through the waveguide. In Fig. 3 we plot the log of this quantity versus the log of the input power. In region I, the total losses that are due to $\mathrm{SH}$ generation are small, and therefore the throughput power is linear in the input power. This is the regime in which Cerenkov SHG experiments have been performed to date and in which theoretical analyses have been valid., ${ }^{3,9}$

In region II optical limiting of the fundamental is clearly observed. In fact the output evolves into a plateau that is flat to within $\pm 8 \%$ over more than three decades of input power. The observed behavior can be understood qualitatively: Because the conversion of the fundamental wave into the leaky waves at the $\mathrm{SH}$ increases with input power and because this converted power does not return to the fundamental (because it is radiated out of the waveguide core by the leaky modes), the losses that the fundamental wave incurs during propagation increase with input power. This behavior is strongly reminiscent of the effects of twophoton absorption, and this is indeed a good analogy because SHG in this regime is also an intensitydependent effect. ${ }^{10}$ The advantage of an optical power limiter based on Cerenkov SHG is that the power of the incoming wave does not have to be dissipated inside the limiting device. Instead it is converted into the harmonic frequency and subsequently can be dealt with in an appropriate way. Therefore the power that the device can handle is limited not by its dissipation capacity but rather by the intensity damage threshold of the nonlinear material. For the structure investigated the input-power threshold for limiting is $\sim 2 \mathrm{MW} / \mathrm{m}$, corresponding to only $6.4 \mathrm{~W}$ in the equivalent fiber geometry. Because of large losses of the leaky modes the acceptance bandwidth of these structures can be quite large when compared with the tight phase-matching constraints encountered in SHG involving guided modes only.
We believe that the most fascinating region is region III, in which unexpected physics is found. Note that after the fundamental throughput switches back to almost unity, there is very little SHG radiated into the cladding. (It occurs only immeidately at the entrance face because of the boundary conditions on the $\mathrm{SH}$.) The $\mathrm{SH}$ is now coguided along with the fundamental. The corresponding transverse distributions of the fundamental and the $\mathrm{SH}$ fields are shown in Fig. 4, along with those in regions I and II for comparison. Whereas in regions I and II the SH field exhibits a strong tail into the cladding, the radiated SHG field in region III is totally confined to the nonlinear film. Such changes cannot be analyzed with a coupled-mode treatment in which the modal field profiles are specifically assumed not to change with power. Furthermore, the fundamental is also more confined to the film than in regions I and II. This is reminiscent of the recently discovered solitary waves in $\chi^{(2)}$ media in which the fundamental and the harmonic are strongly coupled and propagate without diffracting in space. ${ }^{11}$ For high enough fundamental input intensities, the two beams can be locked together, even in the presence of walk-off, and at high intensities the $\mathrm{SH}$ becomes

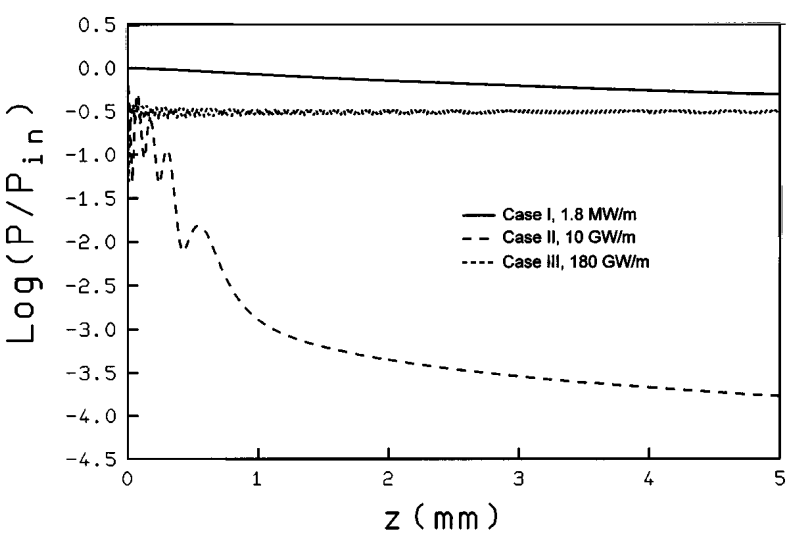

Fig. 2. Logarithm of the transmission of the $\mathrm{TE}_{0}(\omega)$ mode as a function of the propagation distance for the three different input powers.

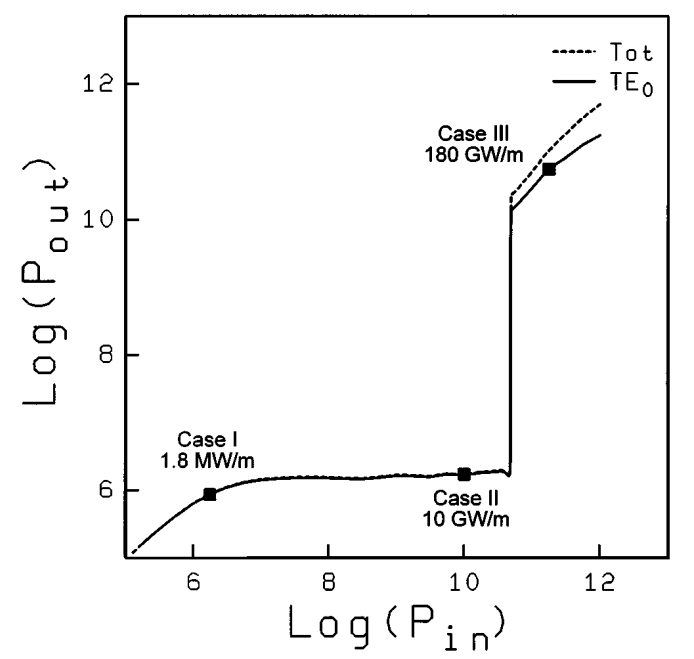

Fig. 3. Output power of the guided fundamental mode (solid curve) and total power (dashed curve) versus input power. 


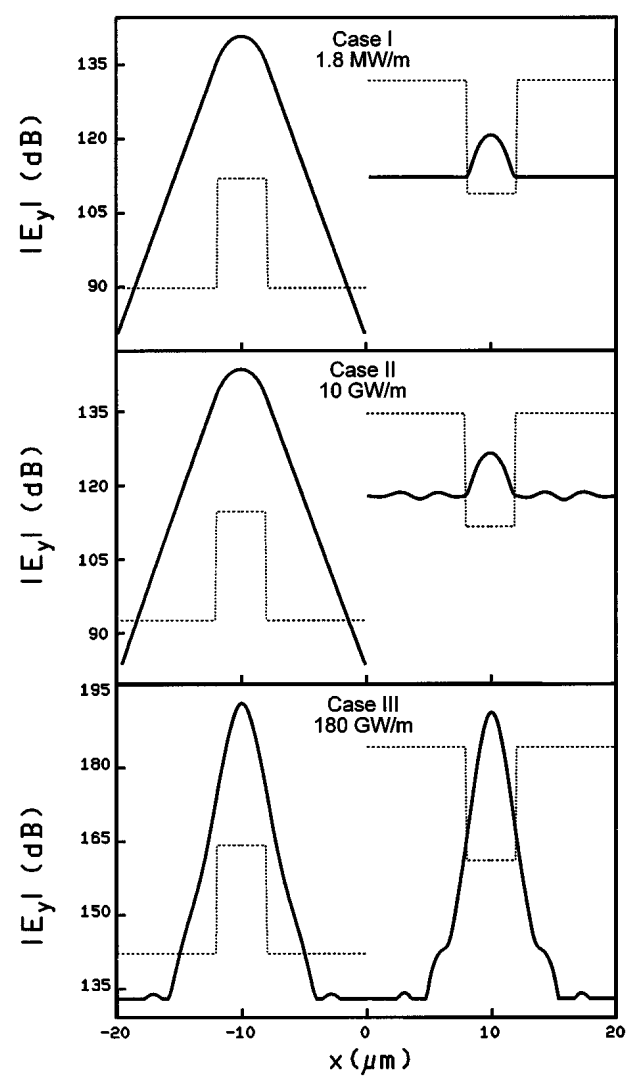

Fig. 4. Logarithm of the field profiles of the fundamental (left) and SH (right) fields after $5-\mathrm{mm}$ propagation for cases I (top), II (middle), and III (bottom). The dashed lines represent the refractive-index profiles (not scaled).

locked to the fundamental in the waveguide. Indeed, solitary waves have been observed in which diffraction is defeated by the combined effects of cascaded selffocusing and parametric gain guiding. Here, diffraction and the nonguiding of the harmonic inferred by $n_{\mathrm{c} 1}(2 \omega)>n_{c}(2 \omega)$ are defeated by the same effects. As a result, Čerenkov SHG is actually inhibited.

This effect clearly produces a switch with a very sharp threshold. The transmission change is $\sim 10^{4}$ for an intensity increase of less than $2 \%$. However, this corresponds to an intensity $>10^{12} \mathrm{~W} / \mathrm{cm}^{2}$, which exceeds the damage threshold of most dense, transparent optical materials. We note, however, that the switching intensity is proportional to $\left|\chi^{(2)}\right|^{-2}$ and hence can be reduced in some existing nonlinear materials. For example, 4-(4-dimethylamino styrene)methylpyridinium tosylase (DAST) has a reported nonlinearity of $400 \mathrm{pm} / \mathrm{V} .^{12}$

In summary, SHG that is due to Čerenkov radiation into a waveguide cladding exhibits interesting phenomena beyond the nondepletion approximation. The fundamental throughput first reaches a plateau and then becomes independent of increasing input power.
It then abruptly increases by orders of magnitude, approaching the input fundamental power, at which point solitary waves are formed because of strongly coupled fundamental and harmonic waves. At this point, the Čerenkov SHG effectively disappears.

The research of G. Krijnen has been made possible by a fellowship of the Royal Netherlands Academy of Arts and Sciences. The research at the Center for Research and Education in Optics and Lasers was supported by the U.S. Air Force Office of Scientific Research.

\section{References}

1. See Compact Blue-Green Lasers, Vol. 6 of 1992 OSA Technical Digest Series (Optical Society of America, Washington, D.C., 1992).

2. For example, G. I. Stegeman, in Proceedings of Erice Summer School on "Nonlinear Waves in Solid State Physics," A. D. Boardman and T. Twardowski, eds., Vol. 247 of NATO ISI Series (Plenum, London, 1990), p. 463.

3. For example, K. Chikuma and S. Umegaki, J. Opt. Soc. Am. B 9, 1083 (1992); T. Suhara, T. Morimoto, and H. Nishihara, IEEE J. Quantum Electron. 29, 525 (1993).

4. G. J. M. Krijnen, H. J. W. M. Hoekstra, and P. Lambeck, presented at the European Conference on Integrated Optics, Ville de Neuchatel, Neuchatel, Switzerland, April 18-23, 1993.

5. G. J. M. Krijnen, W. Torruellas, G. I. Stegeman, H. J. W. M. Hoekstra, and P. V. Lambeck, in GuidedWave Optoelectronics: Device Characterization, Analysis, and Design, T. Tamir, H. Bertoni, and G. Griffel, eds. (Plenum, New York, 1995), p. 381.

6. W. E. Torruellas, G. J. M. Krijnen, D. Y. Kim, R. Schiek, G. I. Stegeman, P. Vidakovic, and J. Zyss, Opt. Commun. 112, 122 (1994).

7. G. J. M. Krijnen, W. Torruellas, G. I. Stegeman, H. J. W. M. Hoekstra, and P. V. Lambeck, IEEE J. Quantum Electron. 32, 729 (1996).

8. D. Y. Kim, W. E. Torruellas, J. Kang, C. Bosshard, P. Vidakovic, J. Zyss, W. Moerner, R. Twieg, and G. Bjorklund, Opt. Lett. 19, 868 (1994).

9. For example, M. J. Li, M. DeMicheli, Q. He, and D. B. Ostrowsky, IEEE J. Quantum Electron. 26, 1384 (1990); H. Sato, Y. Azumai, and H. Nozawa, Opt. Lett. 19, 93 (1994).

10. E. W. Van Stryland, H. Vanherzeele, M. A. Woodall, M. J. Soileau, A. L. Smirl, S. Guha, and T. F. Boggess, Opt. Eng. 24, 613 (1985).

11. W. E. Torruellas, Z. Wang, D. J. Hagan, E. W. Van Stryland, G. I. Stegeman, L. Torner, and C. R. Menyuk, Phys. Rev. Lett. 74, 5036 (1995); R. Schiek, Y. Baek, and G. I. Stegeman, "One-dimensional spatial solitons due to cascaded second-order nonlinearities in planar waveguides," Phys. Rev. A (to be published).

12. C. Bosshard, G. Knopfle, P. Pretre, S. Follonier, C. Serbutoviez, and P. Gunter, Opt. Eng. 34, 1951 (1995). 